BIOCYT Biología, Ciencia y Tecnología, 12(45): 834-845, 2019 (enero-marzo)

ISSN: 2007-2082

http://revistas.unam.mx/index.php/biocyt

Publicada en la Facultad de Estudios Superiores Iztacala, Universidad Nacional Autónoma de México

\title{
COMPARACIÓN DE LA DIETA DEL CACOMIXTLE NORTEÑO, BASSARISCUS ASTUTUS DE UN BOSQUE TEMPLADO Y UN MATORRAL XERÓFILO, DEL CENTRO DE MÉXICO
}

\section{COMPARISON OF THE DIET OF THE RINGTAIL, BASSARISCUS ASTUTUS FROM A TEMPERATE FOREST AND A XEROPHYTHIC SCRUB, FROM CENTRAL MEXICO}

\author{
Gina Elizabet Rosina Castillo-Picazo ${ }^{1,1}$ y Rodolfo García-Collazo ${ }^{1,2} \bowtie \triangle$ \\ 1Laboratorio de Zoología. FES Iztacala UNAM. Av. de los Barrios No. 1 Tlalnepantla de Baz, Estado de México. \\ México CP 54090. 1,1 gina.castillo@conanp.gob.mx 1,2® $\underline{\text { collazo@unam.mx }}$
}

\begin{abstract}
The present study aims to deepen on the diet of the Ringtail (Bassariscus astutus) by analyzing their scats in two locations, one in an Oak forest in Mexico State (AST) and other in a xerophytic scrub in Hidalgo State (TDR). A 67 scats were analyzed, obtained in 2007. The VIA was measured taking into account the following data: weight and occurrence frequency of the food items. In AST 23 items were consumed and in TDR 21 items. The primary food items in AST were the nopal fruits Opuntia streptacantha and Opuntia megacantha, Melolonthidae beetles, also the consumption of waste from food of anthropogenic origin, this may have negative consequences in the organism's survival. In TDR the fruits Myrtillocactus geometrizans, the rat Sigmodon hispidus and the Melolonthidae beetles preferentially. There was a significant difference in the consumed of items between seasons inside each location and between both locations. The trophic diversity is higher in the dry season in both locations; between locations the value of diversity was similar. B. astutus had an omnivore diet; generalist and opportunist.
\end{abstract}

Key Words: Bassariscus astutus, diet, oak forest, xerophytic scrub, México.

Manuscrito recibido el 30 de diciembre de 2017, aceptado el 20 de noviembre de 2018. 


\section{RESUMEN}

El presente estudio buscó profundizar en el conocimiento de la alimentación del cacomixtle norteño (Bassariscus astutus) mediante el análisis de sus excretas en dos localidades, una en un bosque de encino en el Estado de México (AST) y otra en un matorral xerófilo en Hidalgo (TDR). Se analizaron 67 excretas, obtenidas en 2007. Con el peso y frecuencia de ocurrencia de las presas consumidas se calculó el Valor de Importancia Alimentaria para conocer la importancia de cada presa. En AST se consumió 23 presas y en TDR 21 presas. Los elementos más importantes en la dieta en AST fueron los frutos del nopal Opuntia streptacantha y Opuntia megacantha, escarabajos Melolonthidae, además se presentó el consumo de desechos de alimentos de origen antropogénico que pueden tener consecuencias negativas en los organismos. Mientras que en TDR fueron los frutos de Myrtillocactus geometrizans, la rata Sigmodon hispidus y los escarabajos Melolonthidae. Existieron diferencias significativas en las presas consumidas entre temporadas en cada localidad y entre localidades. La diversidad trófica fue mayor en la temporada seca en ambas localidades, entre localidades fue similar el valor de la diversidad. B. astutus presentó una dieta omnívora, generalista y oportunista.

Palabras clave: Bassariscus astutus, dieta, bosque templado, matorral xerófilo, México.

\section{INTRODUCCIÓN}

El conocimiento de la alimentación es uno de los aspectos para el conocimiento de la ecología de las especies animales, ya que de los patrones de utilización de los recursos alimentarios dependerá el uso del hábitat, la conducta de la especie y la dinámica de sus poblaciones entre otros conocimientos. Este tipo de estudios evidencian una serie de relaciones ecológicas que de otra forma serían imperceptibles y que son de importancia para el manejo de las poblaciones naturales (Acosta, 1982; Denis, 2000). El cacomixtle norteño (Bassariscus astutus Lichtenstein, 1830) es encontrado en regiones templadas, áridas y tropicales de Norteamérica y hasta Centroamérica. En México habita prácticamente en todo el país, excepto en la península de Yucatán y Chiapas (Ceballos y Galindo, 1984; Aranda, 2000; Ceballos y Oliva, 2005).

Estudios dirigidos para conocer la dieta del cacomixtle norteño en ambientes templados de Texas muestran como componentes principales materia vegetal, roedores, aves (Passeriformes), insectos y arañas (Toweill y Teer, 1977). En el estado de Nuevo León, México, se reportó el consumo de materia vegetal, insectos, mamíferos, reptiles, aves, arácnidos y miriápodos (González, 1982). En un área de matorral xerófilo del estado de Hidalgo, los componentes en la dieta del cacomixtle, en la época seca fueron, frutos de Opuntia cantabrigiensis, insectos, el roedor Peromyscus sp. y aves; mientras que en la época de lluvias consumió frutos de Myrtillocactus geometrizans, insectos, aves y roedores del género Peromyscus sp. (Nava-Vargas et al., 1999). En la reserva ecológica "El Pedregal de San Ángel" con vegetación de matorral xerófilo y cercano a un ambiente suburbano, Castellanos (2006) encontró que los elementos de origen animal y vegetal tienen la misma importancia en su alimentación, también encontró evidencia de consumo de alimentos de origen antropogénico. En otro matorral xerófilo, en la isla San José ubicada en el golfo de California, Rodríguez-Estrella et al. (2000) encontraron que el cacomixtle se alimenta de ortópteros, escarabajos de la familia Tenebrionidae, escorpiones, del ratón Chaetodipus spinatus, la rata Neotoma lepida y en menor grado de los frutos de Phaulothamnus spinecens también con evidencias del consumo de carroña. 
En una población de cacomixtle que habita el bosque tropical caducifolio y el bosque templado en la sierra del Carmen en el Estado de México, predominó el consumo de los componentes animales en la época de lluvias y en la temporada seca, sobresalieron insectos de la familia Acrididae, roedores y frutos de Solanaceae (Morales, 1998). Pese a la amplia distribución de B. astutus en diferentes ambientes, son escasos los estudios que se han realizado en zonas de bosque templado y de matorral xerófilo, que son dos ambientes diferentes pero cercanos en distancia, esto despierta el interés de conocer más acerca de la dieta de la especie en ambos tipos de vegetación, para conocer y entender el papel que desempeña en el ecosistema. El objetivo del presente estudio fue conocer la dieta de B. astutus (cacomixtle) con base en el análisis de sus excretas, así como realizar una comparación de elementos consumidos en una zona de bosque templado en el Estado de México y en otra zona de matorral xerófilo en el estado de Hidalgo.

\section{MATERIALES T MÉTODOS}

\section{Área de estudio}

La primera localidad se encuentra en Arcos del Sitio, en el municipio de Tepotzotlán, en el Estado de México (AST). Comprende una superficie de 75 ha. dentro de la zona ejidal de San José Piedra Gorda dentro de los límites del parque estatal "Sierra de Tepotzotlán", sus coordenadas geográficas son $19^{\circ} 45^{\prime} 55.9^{\prime \prime}$ latitud $\mathrm{N}$ y $99^{\circ} 20^{\prime} 18.6^{\prime \prime}$ longitud $\mathrm{O}$ y una altitud de 2373 metros sobre el nivel del mar (msnm) (Gobierno del Estado de México, 2001). La vegetación predominante es el bosque de encino, representado por Quercus rugosa, Quercus. laeta y Quercus mexicana, asociado a Arbutus xalapensis (madroño) y Alnus arguta (aile), Mulhembergia cordata y Mulhembergia sessiflora (zacatones) y diversas especies de las familias Compositae, Labiatae, Gramíneae y Leguminoseae; también existe matorral xerófilo, representado por O. streptacantha y Opuntia megacantha (nopales), así como Acacia farnesiana (huizache), Acacia filicoides, Prosopis juliflora (mezquite) y Eysenhartia polystachia (palo dulce) y pastizal (Gobierno del Estado de México, 2001). Prevalece un clima templado subhúmedo $\mathrm{C}(\mathrm{wO})(\mathrm{w})\left(\mathrm{i}^{\prime}\right) \mathrm{g}$, siendo el más seco de los templados con lluvias en verano (García, 2004). La temperatura media anual fluctúa entre 9.4 y $14.4^{\circ} \mathrm{C}$. Las lluvias de origen ciclónico se presentan durante el verano y en menor proporción se asocian a nortes en el invierno. La estación lluviosa se encuentra entre los meses de mayo a octubre. La precipitación pluvial oscila entre 600 y 700 mm. (Gobierno del Estado de México, 2006).

La segunda localidad se encuentra en el municipio de Tepeji del Río de Ocampo, estado de Hidalgo (TDR), el área de estudio tiene una superficie de $364.5 \mathrm{~km}^{2}$, las coordenadas geográficas extremas son latitud máxima $20^{\circ} 00^{\prime} 52.04^{\prime \prime} \mathrm{N}$ y latitud mínima $19^{\circ} 46^{\prime} 13.97^{\prime \prime} \mathrm{N}$; longitud máxima $99^{\circ} 29^{\prime} 00^{\prime \prime}$ O y longitud mínima 99 46'13.49" O. Se encuentra a $2150 \mathrm{msnm}$, (Gobierno del Estado de Hidalgo, 2004). La vegetación se compone de matorral xerófilo y bosque de encino, con componentes de los géneros Opuntia (Opunta streptacantha, Opuntia megacantha), M. geometrizans (garambullo), Prosopis (mezquite), Acacia (huizache), Fraxinus (fresno) y Quercus (encino), y extensiones de pastizales inducidos (Gobierno del estado de Hidalgo, 2004). Se presenta un clima semi-seco templado $\mathrm{Bs}(\mathrm{w})(\mathrm{w})\left(\mathrm{i}^{\prime}\right) \mathrm{g}$ (García 2004). Su temperatura mensual oscila entre 12 y $18^{\circ} \mathrm{C}$. La temporada de lluvias comprende de junio y julio, siendo los de mayor precipitación pluvial y diciembre y febrero los de menor precipitación, temporada seca. El nivel promedio de precipitación es de $704.5 \mathrm{~mm}$ (Gobierno del estado de Hidalgo, 2004).

Se recolectaron excretas en muestreos mensuales de febrero a septiembre de 2007, las cuales fueron confirmadas in situ con base en sus características morfológicas de acuerdo a Aranda (2000). Para tener muestras de referencia y efectuar la comparación, se realizó la captura de roedores con trampas Sherman cebadas con hojuelas de avena. Los organismos capturados se determinaron a nivel de especie (Ceballos y Oliva, 2005), las que también fueron confirmadas con el análisis de los 
pelos de guardia (Arita, 1985). Cada excreta fue tratada como una muestra independiente, se secaron a $60^{\circ} \mathrm{C}$, posteriormente fueron lavadas con detergente para disgregar y separar grasas (Parker, 1986). Se separaron los componentes. El siguiente paso fue la determinación de cada uno de los componentes presa. La determinación de mamíferos se basó en la caracterización del pelo encontrado en las heces y que son elementos confiables para identificar las presas consumidas (Baca y Sánchez-Cordero, 2004). Se usó la técnica de preparados fijos de los pelos modificada de Arita (1985) y de Monroy-Vilchis y Rubio (2003), las claves de estos mismos autores fueron empleadas para la determinación taxonómica. Se utilizaron los caracteres microscópicos del pelo: tales como el diámetro, tipo de médula y diámetro, todo el material examinado fue fotografiado, las mediciones se realizaron con en el software Image Tool $1.27( \pm 0.05)$. Las preparaciones de los pelos obtenidos de las excretas de este estudio fueron comparadas con las de la colección de Arita (1985), que se encuentra resguardada en el Laboratorio de Microcine de la Facultad de Ciencias de la UNAM, para confirmar la correcta determinación taxonómica.

Para el caso de los insectos se agruparon alas, apéndices, segmentos del tórax, mandíbulas y cabeza, para formar organismos completos y con ello poder contabilizarlos e identificarlos (Lehmkuhl, 1979; Borror et al., 1989) hasta el nivel taxonómico posible. Los vegetales consumidos se compararon con muestras observadas in vivo durante los muestreos y confirmados con ejemplares depositados en el herbario de la FES Iztacala, UNAM. La determinación de las semillas se llevó acabo con el apoyo de la colección del Herbario de la Escuela Nacional de Antropología e Historia. Algunas de las muestras de los especímenes colectados se depositaron en ambas colecciones. Para el caso de las aves, no fue posible su identificación taxonómica hasta género y especie, con base al plumaje por ello se determinaron como Aves.

Posterior a la identificación de las diferentes presas y el registro de su respectivo peso, se calculó el Valor de Importancia Alimenticia (VIA) de cada tipo de presa, con la formula modificada de Acosta (1982), para examinar la suma de dos componentes en el análisis de dietas: a) Peso Porcentual: porcentaje en peso (g) que representa cada elemento presa con respecto al total, 2) Frecuencia Relativa de Ocurrencia: el número de excretas en que aparece un determinado elemento presa, con respecto al total de excretas analizadas.

VIA $=V^{\prime} i j+F i j \quad$ Dónde: $V^{\prime} i j=V i j / \sum V i j ~ y, \quad F i j=F i j / ~ N j$

$\mathrm{Vij}=$ Peso de cada presa diferente.

$\sum \mathrm{Vij}=$ Sumatoria del peso total de las especies presa identificadas en las excretas.

Fij = Frecuencia de ocurrencia o número de excretas donde se presenta cada especie presa.

$\mathrm{Nj}=$ Número total de excretas analizadas.

El intervalo de VIA toma valores de 0 a 2, significando que mientras más se acerca un valor a cero, las presas son poco consumidas, mientras que cuando el valor se acerca a dos, las presas son más consumidas. Esta fórmula es adecuada ya que usa la suma de ambos parámetros, reflejando el efecto conjunto del volumen y la frecuencia relativa de ocurrencia. En contraste con los estudios que analizan de manera individual el volumen de consumo o únicamente la frecuencia de ocurrencia. Se aplicó una prueba de Chi cuadrada $\left(\mathrm{X}^{2}\right)$ para evaluar las posibles diferencias en los VIA de las presas entre épocas del año (secas VS lluvias) por localidad y entre ambas localidades. Para conocer la diversidad de la dieta entre las temporadas de lluvias y seca y entre localidades se utilizó el índice de Shannon Weaver $\left(\mathrm{H}^{\prime}\right)$ (Krebs, 1989) y el valor de equitatividad de Pielou (J'). 


\section{RESULTADOS}

Se analizaron 67 excretas de B. astutus de ambas localidades. El número de tipos de presas identificadas que conformaron la dieta del cacomixtle fue de $\mathrm{n}_{\mathrm{AST}}=23$ (Fig. 1) y de $\mathrm{n}_{\mathrm{TDR}}=21$ (Fig. 2). Con base en los porcentajes de ocurrencia por grupos superiores taxonómicos (Tabla 1), en cada localidad, en ambas temporadas (seca y lluvias), se observa que los componentes vegetales presentan un valor similar de ocurrencia $(47 \%)$ a la suma de los componentes animales: artrópodos, mamíferos y aves (49\%). Dentro de los componentes animales los artrópodos superan a los mamíferos y aves en ambas localidades (tabla 1). En el caso exclusivo del bosque templado (AST) se registró el consumo de desechos de origen antropogénico (papel servilleta, plástico, papel aluminio y semillas de pepino Cucumis sativus).

Tabla 1. Porcentaje de ocurrencia (\%) anual para cada grupo taxonómico superior, de alimento registrado en 67 excretas de B. astutus.

\begin{tabular}{ccc}
$\begin{array}{c}\text { Presas consumidas por } \\
\text { grupo taxonómico }\end{array}$ & $\begin{array}{c}\text { Bosque templado (AST) } \\
(\mathrm{n}=37)\end{array}$ & $\begin{array}{c}\text { Matorral xerófilo (TDR) } \\
(\mathrm{n}=30)\end{array}$ \\
\hline vegetales & 47 & 51 \\
artrópodos & 32 & 29 \\
mamíferos & 9 & 16 \\
aves & 6 & 5 \\
desechos antropogénicos & 5 & \\
reptil & 2 & 100 \\
Total & 100 &
\end{tabular}

En la localidad AST se analizaron 37 excretas dentro de la cuales se identificaron un total de 23 elementos presa diferentes en la dieta del cacomixtle (Fig. 1). El espectro de presas consumidas incluye elementos vegetales tales como frutos de Mammillaria magnimmama, O. streptacantha, O. megacantha, Phytolacca icosandra, Prunus serothina, Quercus sp., Smilax sp., Geranium sp., Gramineae y Solanaceae. Del grupo animal los roedores: Oryzomis couesi, Reithrodontomys sumichtrasi, Sciurus aureogaster y roedores no identificados; aves e insectos de las familias: Acrididae, Apidae, Formicidae y Melolonthidae; del orden coleóptera no identificados; a nivel de Clase: aves y el reptil: Sceloporus torquatus. El registro de desechos de origen antropogénico (servilletas de papel, bolsas de plástico, trozos de papel aluminio y semillas de pepino) y lo que no fue posible determinar se registró como Materia Vegetal no identificada (MVNI) y Materia Orgánica no identificada (MONI).

En los VIA estacionales se encontraron diferencias significativas en los componentes presa entre ambas temporadas del año $\left(X^{2}=3.2 ;\right.$ g.l.; $\left.p<0.05\right)$. Fueron los frutos de $O$. streptacantha los que presentaron la mayor importancia en ambas épocas, sobre todo en lluvias (VIA $\mathrm{AST}=1.25$ ), en la figura 1 se pueden ver los valores de VIA para cada presa. Los artrópodos también mostraron un valor importante en la dieta, el consumo de las presas varió con la época, y los más consumidos fueron los escarabajos Melolonthidae $\left(\mathrm{VIA}_{\text {lluvia }}=0.36\right.$ y $\mathrm{VIA}_{\text {secas }}=0.33$ ) y las hormigas Formicidae $\left(V_{\text {VIA }}\right.$ secas $\left.=0.2\right)$. El consumo de mamíferos, que fueron en su mayoría roedores, fue más bajo que el de otras presas, sobresale $O$. couesi por su mayor consumo entre los roedores y su presencia en ambas épocas del año $\left(\mathrm{VIA}_{\text {lluvia }}=0.15\right.$ y VIA $\left.\mathrm{V}_{\text {secas }}=0.07\right)$. Los desechos antropogénicos, únicamente fueron registrados en la época de secas $\left(\mathrm{VIA}_{\text {secas }}=0.3\right)$. 
La diversidad trófica $\left(\mathrm{H}^{\prime}\right)$ de los componentes alimentarios en AST fue mayor en la temporada seca (2.81) respecto a la de lluvias (1.97). La equitatividad fue similar entre ambas $\left(\mathrm{J}^{\prime}=\right.$ 0.64 y 0.65 respectivamente).

De la localidad TDR se analizaron 30 excretas, encontrándose un total de 21 elementos presa diferentes (Fig. 2). El espectro alimentario se conformó por elementos vegetales: Mammillaria magnimamma, Myrtillocactus geometrizans, O. streptacantha, O. megacantha, P. serothina, Simpsia amplexicaulis, Passiflora sp., Solanum sp., Ipomoea sp., Graminae; del grupo de los animales fueron los roedores: Sigmodon hispidus, Sphermophilus variegatus P. maniculatus, P. difficilis, y un Rodentia no identificado; insectos de las familias: Melolonthidae, Acrididae, Vespidae, Formicidae e insectos de Coleóptera no identificados. Además de la clase Aves, MVNI y MONI.

Al comparar el Valor de Importancia Alimentaria de las presas entre ambas temporadas del año en TDR (Fig. 2), la prueba de Chi cuadrada mostró que existen diferencias significativas $\left(\mathrm{X}^{2}\right.$ =10.56; g.l. 20; $p$ < 0.05). Sobresale el mayor valor de importancia alimentaria de los frutos de $M$. geometrizans $\left(\mathrm{VIA}_{\text {lluvia }}=1.21\right)$ y frutos de O. streptacantha $\left(\mathrm{VIA}_{\text {secas }}=0.54\right)$ entre los componentes vegetales (Fig. 2). Seguido por los roedores $S$. hispidus $\left(\mathrm{VIA}_{\text {secas }}=0.98\right)$ y $S$. variegatus $\left(\mathrm{VIA}_{\text {secas }}=0.42\right)$; y del grupo de los artrópodos los escarabajos Melolonthidae $\left(\mathrm{VIA}_{\text {lluvias }}=0.46\right)$ y Acrididae $\left(\mathrm{VIA}_{\text {secas }}\right.$ $\left.=0.41 \mathrm{y} \mathrm{VIA}_{\text {lluvia }}=0.15\right)$. Con respecto a la diversidad trófica el valor fue mayor y representativo en la temporada seca (2.6) con respecto a la época de lluvias (1.9). La equitatividad fue más elevada en la seca que en la de lluvias $\left(\mathrm{J}^{\prime}=0.66 \mathrm{y} \mathrm{J}^{\prime}=0.51\right.$ respectivamente $)$.

El espectro alimentario se conforma en ambas localidades por los mismos grupos de alimento: frutos de cactáceas, roedores, artrópodos y aves. En AST, además se presentó, un reptil y materia proveniente de desechos de alimentos de origen humano (Fig. 1).

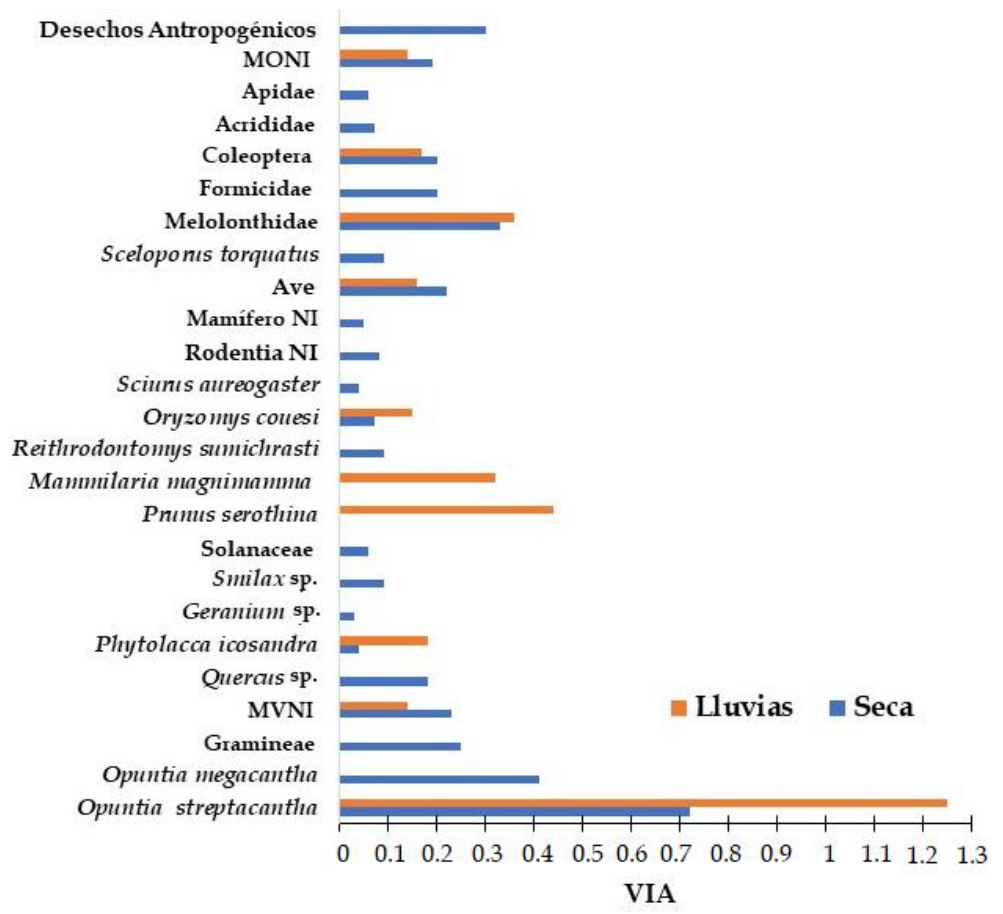

Fig. 1. Comparación de los valores de importancia alimentaria (VIA) de todas las categorías presa registradas en B. astutus en bosque templado (Arcos del Sitio), en dos temporadas del año. 
Del componente vegetal, los elementos más importantes fueron los frutos del nopal cardón O. streptacantha y O. megacantha en AST. Mientras que, O. streptacantha y M. geometrizans lo fueron en TDR. Se observó diferencia importante entre ambas localidades en el consumo de mamíferos, ya que no se observaron especies en común, además los valores de consumo fueron mayores en TDR. En ambas localidades existió consumo importante de artrópodos principalmente de las familias Melolonthidae, Formicidae y Acrididae. Fueron significativas las diferencias en el consumo de los componentes presa entre ASR y TDR $\left(X^{2}=7.98\right.$; g.l. 32; $\left.p<0.05\right)$. En lo que respecta a la diversidad trófica $\left(\mathrm{H}^{\prime}\right)$ en TDR resulto ser ligeramente mayor (2.8) con respecto a AST (2.6), aunque la equitatividad es mayor en TDR $\left(\mathrm{J}^{\prime}=0.65\right)$ que en AST $\left(\mathrm{J}^{\prime}=0.58\right)$.

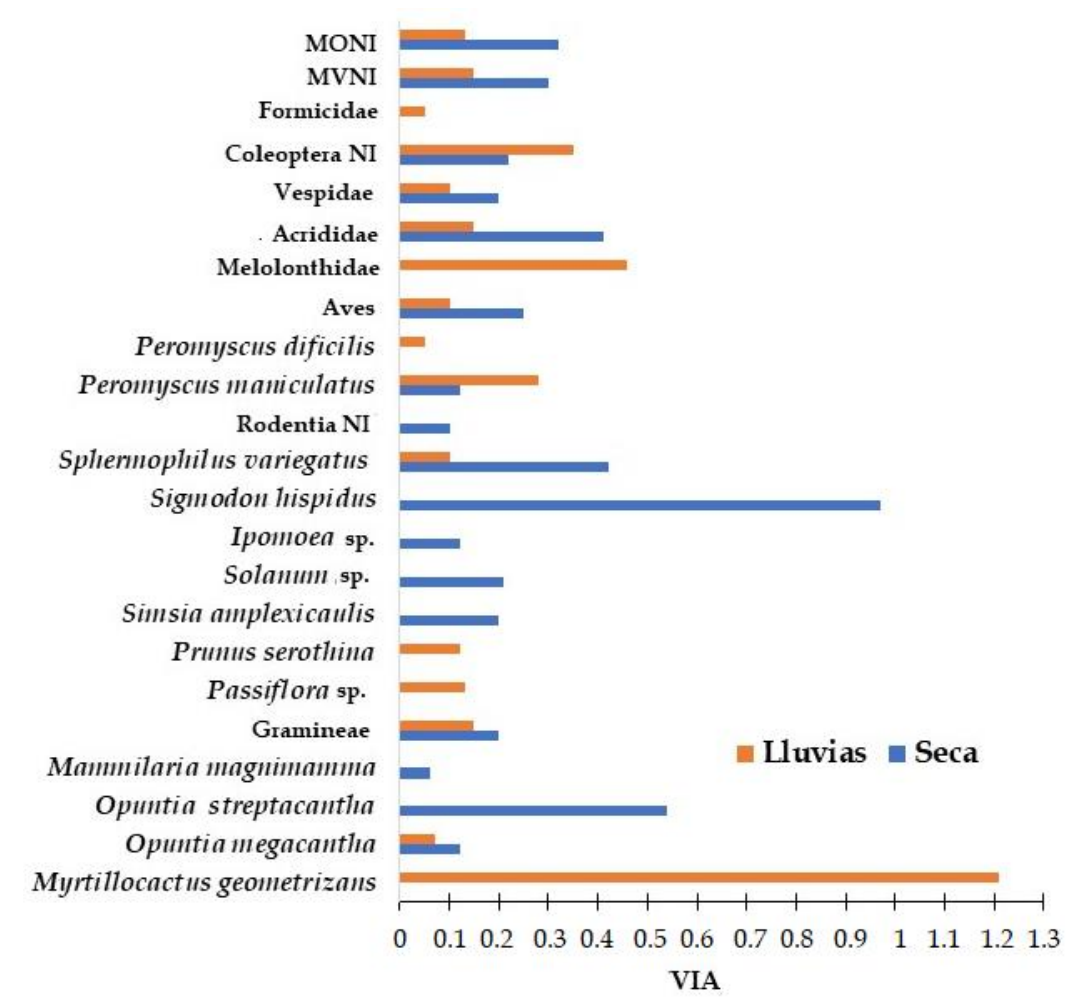

Fig. 2. Comparación de los valores de importancia alimentaria (VIA) de todas las categorías presa registradas en B. astutus en matorral xerófilo (Tepeji del Río), en dos temporadas del año.

\section{DISCUSIÓN}

En contraste con otros estudios realizados en bosque templado (Taylor, 1954; Toweill y Teer, 1977; Morales, 1998) y matorral xerófilo (Rodríguez-Estrella et al., 2000) en los cuales el componente animal, invertebrados y vertebrados, fue el grupo presa consumidas más importantes en la dieta de $B$. astutus a lo largo del año, en el presente estudio los vegetales fueron los más relevantes en el bosque templado (AST) y en el matorral xerófilo (TDR) lo que es igual a lo reportado por Nava-Vargas et al., (1999). En el presente estudio los mamíferos quedaron por debajo del consumo de artrópodos que fueron en su mayoría insectos, mientras que, las aves junto con los reptiles constituyeron un mínimo en el consumo. 
El consumo de frutos de Opuntia y los artrópodos constituyeron los elementos con mayor VIA en ambas localidades. Entre los vegetales, los elementos más importantes fueron los frutos de nopal O. streptacantha (nopal cardón) y O. megacantha (nopal blanco) en AST, mientras que $O$. streptacantha y M. geometrizans (garambullos) lo fueron en TDR. Esto puede deberse a que estos elementos están más disponibles, además los frutos de cactáceas poseen concentraciones altas de agua, azúcares, minerales y vitaminas (Bravo-Hollis y Sánchez-Mojorada, 1991), que son aprovechados por B. astutus en el periodo de secas. Los frutos de Opuntia fueron constantemente consumidos, siendo el único componente en varias excretas. Los frutos de Opuntia como elemento básico en la dieta del cacomixtle en la temporada seca ya ha sido reportado (Taylor, 1954; Toweill y Teer, 1977; Nava-Vargas et al., 1999).

En ambas localidades, el cacomixtle consumió ocasionalmente algunos alimentos, seguramente por su baja abundancia como es el caso de los frutos de P. serothina, M. magnimamma, P. icosandra, Smilax sp., Passiflora sp., Solanum sp. y S. amplexicaulis. Algunas de estas plantas consumidas como P. icosandra, S. amplexicaulis, Smilax sp. y Passiflora sp. se desarrollan como malezas; flora ruderal y arvense son características de áreas perturbadas (Rzedowski, 1994).

Otro elemento vegetal que aparece en el consumo de ambas localidades fueron los pastos o gramíneas, no hay evidencia de que tengan un valor nutritivo para el cacomixtle por lo que se puede interpretar que probablemente son usadas como laxante y también ingeridas de manera accidental con alguna presa, como los artrópodos y roedores, como ya ha sido reportado (Taylor, 1954; Gould y Shaw, 1992; Aranda, 1995). La ingestión de hojas de Quercus en AST probablemente fue accidental al consumir insectos. Ya que su digestión y asimilación es difícil, por su alto contenido en fibras (Esau, 1985).

Un elemento alimentario importante en ambas localidades lo constituyeron los insectos, con mayor VIA en la dieta en TDR. Los escarabajos de la familia Melolonthidae fueron los más relevantes en AST durante la sequía y se mantuvo su consumo durante la época de lluvias en ambas localidades. Otra presa fueron los Coleoptera, el consumo de estas presas es común en la dieta de B. astutus (Taylor, 1954; Toweill y Teer, 1977; Nava-Vargas et al., 1999; Rodríguez-Estrella et al., 2000). Los insectos acrídidos mostraron un valor bajo en AST en comparación con otros trabajos en bosque templado (Taylor, 1954; Morales, 1998), sin embargo, en TDR tomaron mayor VIA siendo uno de los principales elementos.

Entre ambas localidades, el consumo de mamíferos mostró diferencias ya que no hubo especies presa en común, además de que su consumo con base al VIA fue mayor en el matorral xerófilo (TDR). En AST la presa más importante fue la rata O. couesi y en TDR S. hispidus (rata algodonera) y $S$. variegatus (ardillón de roca). La alta importancia en el consumo de $S$. hispidus concuerda con estudios realizados en Texas (Taylor, 1954; Wood, 1954; Toweill y Teer, 1977).

Las aves, en ambas localidades no presentaron valores relevantes, lo que concuerda con otros estudios (Toweill y Teer, 1977; Morales, 1998; Nava-Vargas et al., 1999; Flores, 2001). Su baja relevancia debe ser porque son presas que requieren mayor esfuerzo para su captura. Por otro lado, el consumo de reptiles fue mínimo; se reporta también con mínima importancia en bosque templado (Taylor, 1954) y en matorral seco (Rodríguez-Estrella et al., 2000). Aunque S. torquatus abunda en TDR (Gómez, 2007) no fue aprovechada posiblemente por la dificultad que implica atraparla y a que son de actividad diurna, mientras que, el cacomixtle es fundamentalmente nocturno. 
En el presente estudio se registraron elementos no reportados en la dieta del cacomixtle, tales como frutos de Smilax sp. (zarzaparrilla), flores de Geranium sp. (geranio) y Apidae (abejas) que si pueden ser elementos nutritivos.

Las marcadas diferencias en los elementos consumidos entre estaciones y entre localidades, son reflejo de la marcada estacionalidad ambiental. Estas diferencias indican la ventaja de B. astutus como omnívoro generalista y su comportamiento como el modelo oportunista, al usar distintos recursos alimentarios ante su abundancia (Schoener, 1971).

En el bosque templado (AST) el cacomixtle ingirió mamíferos en mayor cantidad durante la sequía, estos fueron la ardilla $S$. aureogaster y el roedor $R$. sumichtrasi, que se distribuyen en bosques templados y se reproducen durante la primavera (Ceballos y Oliva, 2005); también se alimentó con $O$. couesi que se reproduce durante todo el año y que al igual que los roedores anteriores, se distribuye en bosque templado. En la época de lluvias solo se presentó el roedor O. couesi (rata arrocera), los datos obtenidos en la presente investigación demostraron que los cacomixtles consumieron en su mayoría frutos.

En TDR S. hispidus (rata algodonera) y S. variegatus (ardillón) fueron las presas más consumidas por B. astutus en la época seca, estos roedores se distribuyen en gran variedad de ambientes, entre ellos el matorral xerófilo y se reproduce todo el año, además son activos en el día y la noche y llegan a formar poblaciones numerosas (Ceballos y Oliva, 2005); en temporada de lluvias continuó el consumo de $S$. variegatus y también del ratón $P$. maniculatus y en menor cantidad $P$. difficilis, ambas especies habitan en matorrales y se reproducen entre los meses de junio y octubre (Ceballos y Oliva, 2005). Lo anterior muestra que los mamíferos siguen siendo un grupo indispensable en su dieta, aunque su importancia no siempre sea la primera. Sin embargo, cabe preguntarse porque ocurrió este cambio de dieta, de manera más notable en AST, en donde la importancia de los mamíferos en la dieta quedó relegado a segundo término, lo cual puede deberse a varios factores como la fragmentación del ecosistema, donde los roedores se encuentran en declive poblacional, otra razón puede ser la dificultad de su captura, o por el tamaño poblacional que presentan debido a un bajo éxito reproductivo, o se trata únicamente de un aprovechamiento oportunista de los recursos con mayor disponibilidad como es el caso de los frutos de cactáceas.

En la temporada de secas en AST, en varias muestras se encontraron, evidencias del consumo de alimentos de origen antropogénico (servilletas, plástico, papel aluminio y semillas de pepino C. sativus). Esto debido a que en AST existe un centro ecoturístico al que asiste mucha gente. El consumo de desechos de la actividad humana ya ha sido reportado en el coyote (Aranda, 1995) y también en B. astutus (Rodríguez-Estrella et al., 2000; Castellanos, 2006). La ingesta de plástico es riesgosa para los organismos ya que provoca sensación de saciedad, y en casos más graves, los animales dejan de alimentarse; así mismo, puede producir oclusión o perforación intestinal y la contaminación con subproductos del petróleo y fenoles que se encuentran en el plástico, con fatales consecuencias en el animal (Moore et al., 2001; Van Franeker et al., 2005; Teuten et al., 2009; Simmonds, 2012).

\section{AGRADECIMIENTOS}

A los doctores Patricia Ramírez B., José Luis Gama, al Mtro. Sergio Stanford C., por sus valiosos comentarios. Al Biol. Mario A. de la Concha por su apoyo en campo y en la determinación de semillas. Al Biol. Antonio Barbero responsable del parque sierra de Tepotzotlán. A los ejidatarios de Sn. Francisco Magu. A la Asociación Ecológica de Madero Santiago Tlahutla. A la Mtra. Ana 
Isabel Bieler A. por su apoyo en la toma de microfotografías. Al Mtro. Alejandro Martínez M. del laboratorio de microcine de la Facultad de Ciencias, UNAM. Al Biol. Javier Hernández del Olmo de la ENAH por su apoyo en la determinación de semillas. Al herbario de la FES Iztacala, UNAM. A Moni, Julieta y Miguel por su apoyo en campo. Y a los revisores anónimos de este trabajo.

\section{REFERENCIAS}

1. Acosta M., 1982. Índice para el estudio del nicho trófico. Ciencias Biológicas. Academia de Ciencias de Cuba, 7: 125-128.

2. Aranda M., 1995. Hábitos alimentarios del coyote (Canis latrans) en la Sierra del Ajusco, México. Acta Zoológica Mexicana (Nueva Serie), 65: 89-99.

3. Aranda M., 2000. Huellas y otros rastros de los mamíferos grandes y medianos de México. CONABIO-Instituto de Ecología, A.C. México, D.F.

4. Arita H.T., 1985. Identificación de los pelos de guardia de los mamíferos del Valle de México. (Tesis Profesional, Facultad de Ciencias, Universidad Nacional Autónoma de México, México).

5. Baca I.I. y V. Sánchez-Cordero, 2004. Catálogo de pelos de guardia dorsal en mamíferos del estado de Oaxaca, México. Anales del Instituto de Biología. UNAM. Serie Zoología, 75(2): 383-437.

6. Borror J.D., A.C. Triplehorn y F.N. Jonson, 1989. An introduction to the study of insects. Saunders Collage Publishing. USA.

7. Bravo-Hollis H. y H. Sánchez-Mejorada, 1991. Las cactáceas de México. Vol. I. Universidad Nacional Autónoma de México, México.

8. Castellanos M.G., 2006. Sobre el ámbito hogareño y los hábitos alimentarios de un carnívoro en un ambiente suburbano. El cacomixtle (Bassariscus astutus) en la reserva ecológica "El Pedregal de San Ángel”. Ciudad Universitaria, México, D.F. (Tesis Profesional, Facultad de Ciencias. Universidad Nacional Autónoma de México, México).

9. Ceballos G.G. y C. Galindo, 1984. "Mamíferos silvestres de la Cuenca de México". Limusa. México.

10. Ceballos G.G. y G. Oliva, 2005. Los mamíferos silvestres de México. CONABIO- Fondo de Cultura Económica. México.

11. Denis A.D., 2000. Aplicación del método Jackknife a un descriptor de la dieta en aves. Revista Biología, 14(2): 126-132.

12. Esau K., 1985. Anatomía Vegetal. Ediciones Omega. España.

13. Flores R.A., 2001. Algunos aspectos alimentarios de los mamíferos medianos en la Reserva de la Biosfera Sierra de Huautla, en el Estado de Morelos (Tesis Profesional, FESI, Universidad Nacional Autónoma de México, México).

14. García E. 2004. Modificaciones al sistema de clasificación climática de Köppen. Serie libros 6 Instituto de Geografía, Universidad Nacional Autónoma de México. 
15. Gobierno del Estado de Hidalgo, 2004. Programa de Ordenamiento Ecológico Territorial, Tepeji del Río de Ocampo. Periódico Oficial del Estado de Hidalgo, Tomo CXXXVII No. 26.

16. Gobierno del Estado de México, 2001. Proyecto de conservación ecológica de la zona metropolitana del valle de México. Gobierno del Estado de México-Secretaría de Ecología, México.

17. Gobierno del Estado de México., 2006. Secretaría de Ecología, Coordinación General de Conservación Ecológica. http:// www.edomex.gob.mx (accesado en noviembre 12, 2017).

18. Gómez M.J., 2007. Contribución al conocimiento de la herpetofauna del municipio de Tepeji del Río de Ocampo, Hidalgo (Tesis Profesional, FESI, Universidad Nacional Autónoma de México, México).

19. González F.N., 1982. Estudios preliminares sobre el cacomixtle (Bassariscus astutus), en el municipio de Agualeguas, Nuevo León. (Tesis Profesional Universidad de Nuevo León. México).

20. Gould W.F. y R.B. Shaw, 1992. Gramíneas. Clasificación sistemática. A.G.T Editor. México.

21. Krebs C.J., 1989. Ecological methodology. Harper Collins Publisher. New York.

22. Lehmkuhl M.D., 1979. How to know the aquatic insects. Wm. C. Brown Company Publishers. Dubuque Iowa USA.

23. Monroy-Vilchis O. y R.R. Rubio, 2003. Guía de identificación de mamíferos terrestres del Estado de México, a través del pelo de guardia. Luminaza. Universidad Autónoma del Estado de México. México.

24. Moore C.J., S.L. Moore, M.L. Leecaster y S.B. Weisberg, 2001. A comparison of plastic and plankton in the North Pacific central gyre. Marine Pollution Bulletin, 42: 1297-1300. DOI: https://doi.org/10.1016/S0025-326X(01)00114-X

25. Morales G.A., 1998. Descripción de la dieta de algunos mamíferos silvestres de la Sierra del Carmen, Estado de México. (Tesis Profesional, FESI, Universidad Nacional Autónoma de México, México).

26. Nava-Vargas V., J.D. Tejero y C.B. Chávez, 1999. Hábitos alimentarios del cacomixtle Bassariscus astutus (Carnívora: Procyonidae) en un matorral xerófilo en Hidalgo, México. Anales del Instituto de Biología. UNAM. Serie Zoología, 70(1): 51-63.

27. Parker G.R., 1986. The seasonal diet of coyotes, Canis latrans in northern New Brunswick. Canadian Field Naturalist, 100: 74-77.

28. Rodríguez-Estrella R., A. Rodríguez-Moreno y K. Grajales-Tam, 2000. Spring diet of the endemic ring-tailed cat (Bassariscus astutus insulicola) population of an island in the Gulf of California, México. Journal of Arid Environments, 44: 241-246. DOI: https://doi.org/10.1006/jare.1999.0579

29. Rzedowski J., 1994, Vegetación de México. Editorial Limusa. México. 
30. Schoener T.W., 1971. Theory of feeding strategies. Annual Review of Ecology and Systematics, 2: 369-404. DOI: https://doi.org/10.1146/annurev.es.02.110171.002101

31. Simmonds M.P., 2012. Cetaceans and Marine Debris: The Great Unknown. Journal of Marine Biology, 2012 Article ID 684279, 8. DOI: http://dx.doi.org/10.1155/2012/684279

32. Taylor P.W., 1954. Food habits and notes on life history of ring-tailed cat in Texas. Journal of Mammalogy, 35(1): 55-63.DOI: https://doi.org/10.2307/1376073

33. Teuten E.L., J.M. Saquing, D.R.U. Knappe, M.A. Barlaz, S. Jonsson, A. Bjorn, S.J. Rowland, R.C. Thompson, T.S. Galloway, R. Yamashita, D. Ochi, Y. Watanuki, C. Moore, P.H. Viet, T.S. Tana, M. Prudente, R. Boonyatumanond, M.P. Zkaria, K. Akkhavong, Y. Ogata, H. Hirai, S. Iwasa, K. Mizukawa, Y. Hagino, A. Imamura, M. Saha y H. Takada, 2009. Transport and release of chemicals from plastics to the environment and to wildlife. Philosophical Transactions of the Royal Society B., 364: 2027-2045. DOI: https://doi.org/10.1098/rstb.2008.0284

34. Toweill E.D. y G.J. Teer, 1977. Food habits on ringtails in the Edwards Plateu Region or Texas. Journal of Mammalogy, 58(4): 660-663.

DOI: $\underline{\text { https://doi.org/10.2307/1380015 }}$

35. Van Franeker J.A., M. Heubeck, K. Fairclough, D.M. Turner, M. Grantam, E.W.M. Stienen, N. Guse, J. Pedersen, K.O. Olsen, P.J. Anderson y B. Olsen, 2005. Save the North Sea. Fulmar Study 2002-2004. A regional pilot project for the Fulmar- Litter. Eco QO in the OSPAR area. Wageninger, Alterra. Alterra-rapport 1162. USA.

36. Wood J.E., 1954. Food habits of furbearers of the upland post oak region in Texas. Journal of Mammalogy, 35(3): 406-414. DOI: 10.2307/1375965 
BIOCYT Biología, Ciencia y Tecnología, se encuentra actualmente indexada en
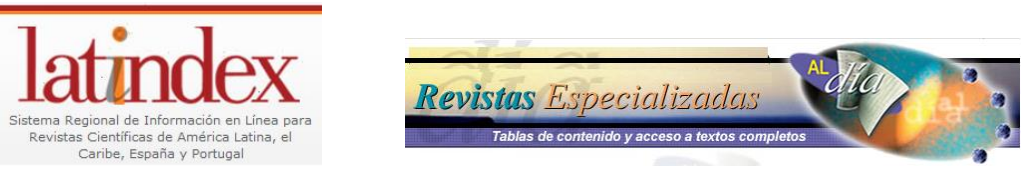

O Dialnet

\section{Actualidad Iberoamericana}

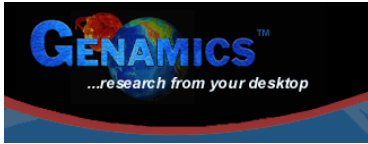

I.Es $\quad$ REMERI $\equiv$
INDI $\mathbf{Z E} \mid \begin{aligned} & \text { de Revistas y } \\ & \text { Publicaciones Periódicas beta }\end{aligned}$

alojada en los repositorios
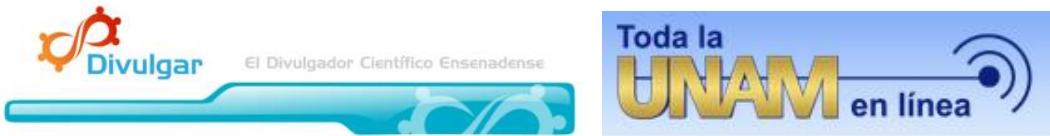

y en bases electrónicas de bibliotecas

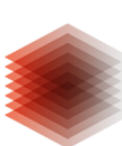

TIB

LEIBNIZ-INFORMATIONSZENTRUM

TECHNIK UND NATURWISSENSCHAFTEN

UNIVERSITÄTSBIBLIOTHEK 